\title{
SIECRIM: Um Sistema para Geração Automática de Relatórios em Segurança Pública
}

\author{
Luiz Alberto Silva ${ }^{3}$, Paula Moreira ${ }^{3}$, Maxwel Macedo Dias ${ }^{1}$, Tácio Ribeiro ${ }^{3}$, \\ Franklim dos Santos ${ }^{2}$, Roberto Cesar Betini ${ }^{4}$, Cleidson de Souza ${ }^{4}$, \\ ${ }^{1}$ Faculdade de Informática - Universidade Federal do Pará (UFPA) \\ ${ }^{2}$ Pós-Graduação em Sistemas de Bancos de Dados (UFPA) \\ ${ }^{3}$ Programa de Pós Graduação em Ciência da Computação (PPGCC) \\ \{xharrisonx, pdanni,taciovinicius\}@gmail.com, \{maxwel_ufpa, \\ lasf_bel\}@yahoo.com.br \\ ${ }^{4}$ Instituto de Ciências Exatas e Naturais - UFPA \\ \{betini, cdesouza\}@ufpa.br
}

\begin{abstract}
This paper presents the Sistema Estatístico Criminal (SIECRIM for short), which has the objective to automate the statistical reports generation, that aids the criminal analysis and strategic actions for the public security. Another peculiar feature from this system is the possibility to integrate the public security's databases.

Resumo. Este trabalho apresenta o Sistema Estatístico Criminal (SIECRIM), cujo objetivo é automatizar a geração de relatórios estatísticos que auxiliam a análise criminal e ações estratégicas. Outra característica peculiar deste sistema é a possibilidade de integração das diferentes bases de dados da Secretaria de Segurança Pública do Estado do Pará.
\end{abstract}

\section{Introdução}

No cenário atual, a informação passou a ser um elemento essencial para o sucesso das organizações. No âmbito comercial, a gerência dos dados traz um diferencial competitivo. Assim empresas do mundo todo estão investindo na busca de melhores respostas e resultados no sentido de verificar a eficácia de suas ações. A informação é importante para que os gestores da segurança possam tomar decisões estratégicas que tragam resultados mais eficientes e satisfatórios para a diminuição da criminalidade.

A informação é um dos principais elementos utilizados na tomada de decisão pelas instituições e advém principalmente das organizações públicas, de seus enormes bancos de dados, que contém informações de contribuintes, estoques, compras, folha de pagamentos, perfil social da população, dentre outros. No entanto, apesar do enorme valor desses dados, a maioria das organizações é incapaz de aproveitar totalmente o que está armazenado em suas bases de dados.

Orlandini (2007) afirma que deve existir um conjunto de características necessárias para que esse "instrumento de trabalho" fundamental realmente atenda às necessidades dos gestores, que são: agilidade - disponível no tempo certo e 
confiabilidade - coesa, correta. E, além disso, precisa ser "certeira", isto é, ágil, confiável e para quem ela realmente será útil.

Os gestores das organizações procuram compreender periodicamente as necessidades e aspirações de grupos de interesse no seu ambiente externo, ou de condições colocadas ao seu funcionamento por esses grupos. Para isto, os gestores são assessorados por profissionais da área da estatística, a fim de construírem relatórios sobre os negócios ou funcionamento das instituições.

Tais relatórios são constituídos basicamente de análise descritivas, tabelas, gráficos e, raramente, mapas. Porém observa-se que as características citadas anteriormente por Orlandini (2007) devem estar presentes nas informações geradas, no entanto, elas demandam num elevado número de profissionais e um excessivo tempo de trabalho.

Nesse sentido, o objetivo deste trabalho será discorrer a respeito de um sistema denominado SIECRIM, que gera relatórios estatísticos automaticamente a partir da base de dados da segurança pública do Estado do Pará. Este relatório é composto de análise descritiva dos dados, construção de tabelas e gráficos e, ainda, de mapas da criminalidade.

Além desta seção introdutória, este artigo apresenta-se na seção 2 uma descrição das estatísticas na área de Segurança Pública; na seção 3 será mostrada a importância da base de dados na realização da análise criminal; na seção 4 será mostrado como o SIECRIM realiza a geração automática de relatórios estatísticos; finalmente, na seção 5 são analisados alguns trabalhos relacionados e finalmente, na seção 6 serão realizadas as considerações finais deste artigo.

\section{Estudo e Análise da Criminalidade}

Na área de Segurança Pública, a produção e divulgação de dados estatísticos sobre crimes, violência e trabalho policial servem, em primeiro lugar, para orientar o governo quanto aos caminhos que se deve seguir no planejamento, execução e re-direcionamento das ações policiais; em segundo, para a população conhecer o que está acontecendo ao seu redor a respeito da criminalidade; e terceiro, para que, conhecendo os fatos criminais e suas áreas de incidência, os diferentes setores da sociedade civil possam objetivar as demandas por providências do poder público e contribuir para o esforço comunitário contra a insegurança.

Os analistas criminais têm o papel de propor melhores formas de gerência das ações estratégicas, e para tanto se utilizam de diferentes informações. De acordo com Steven Gottlieb (2002), a análise criminal pode ser entendida como:

\footnotetext{
"Um conjunto de processos sistemáticos (...) direcionados para o provimento de informação oportuna e pertinente sobre os padrões do crime e suas correlações de tendências, de modo a apoiar as áreas operacional e administrativa no planejamento e distribuição de recursos para a prevenção e supressão de atividades criminais, auxiliando o processo investigativo e aumentando o número de prisões e esclarecimento de casos."
}

Em tal contexto, a análise criminal tem várias funções na organização policial, incluindo a distribuição das viaturas para o patrulhamento, as operações especiais e unidades táticas, investigações, prevenção criminal e serviços administrativos. Nota-se que o fator primordial para o sucesso da análise criminal é a informação de qualidade e no tempo desejado. 
Santos (2002) afirmam que a utilização dos conhecimentos em estatística permitirá ao analista criminal a criação de indicadores que permitam o cruzamento entre informações internas e externas capazes de trazer alguma contribuição para o conhecimento da criminalidade e suas causas.

Acredita-se que a realização de diagnósticos, a definição de critérios de avaliação e a elaboração de medidas de desempenho consistentes são importantes tanto para auxiliar a avaliação da qualidade do trabalho policial, quanto para possibilitar o gerenciamento profissional da polícia. A identificação de padrões, a partir do cruzamento das informações existentes nos bancos de dados das polícias, constitui também uma forma de sistematização que independe da memória individual dos agentes.

Diversos trabalhos científicos têm mostrado a utilização da estatística na área de Segurança Pública. Por exemplo, pode-se citar o trabalho de Ramos et al (2007) que mostra um estudo da série histórica da quantidade de registros de roubos na região metropolitana de Belém, a partir da utilização de uma técnica estatística denominada Análise de Intervenção.

Apesar da importância da estatística para a realização da análise criminal, a geração de relatórios estatísticos pelos funcionários da estatística ainda apresentam algumas limitações e dificuldades, alguns desses problemas serão descritos na próxima subseção.

\subsection{Limitações das Atividades Estatísticas}

Quando se fala sobre geração de relatórios estatísticos da criminalidade, deve-se analisar alguns aspectos e características para prover maiores mecanismos para tomada de decisão e planejamento estratégicos dos gestores da Segurança Pública.

Trabalhar com dados da criminalidade é trabalhar como uma enorme quantidade de dados, que aumentam diariamente. Bancos de dados são medidos atualmente em gigabytes e terabytes. A necessidade de transformar estes terabytes de dados em informações significativas é uma necessidade urgente. Nesse sentido, realizar a confecção de relatórios em um curto espaço de tempo ainda tem sido um desafio, uma vez que tais bases de dados nem sempre chegam às mãos dos estatísticos quando necessário, além disso necessita-se de um grande número de funcionários para conclusão dos relatórios, o que, na maioria das vezes, não se observa nas instituições.

Outro fator é a velocidade no acesso a essa informação, pois o relatório pode ter sido concluído, mas às vezes somente depois de dias ou semanas os gestores têm acesso a essa informação. Este fator é importante, pois o valor da informação depende do momento em que acontece, pois determinado documento pode não ser relevante a uma consulta em determinado momento e tornar a ser em outro [Mizzaro, 1997].

\section{Banco de Dados}

Bancos de dados, (ou bases de dados), são conjuntos de dados compostos por uma estrutura regular que organizam informação. Nesta seção será mostrada a importância das bases de dados para a realização da análise criminal, a necessidade de tratamento nos dados antes da efetiva aplicação das estatísticas e a importância da integração entre as diferentes bases de dados. 


\subsection{A importância da Base de Dados para a realização da Análise Criminal}

Com o passar dos anos, os órgãos de Segurança Pública têm acumulado cada vez mais informações em seus bancos de dados. Como conseqüência, estes bancos de dados passam a conter informações relevantes informando por exemplo como está o perfil e o padrão da criminalidade em uma determinada área.

Tais dados são obtidos, na maioria das vezes, pela polícia civil através dos boletins de ocorrência e pela polícia militar, através dos sistemas de atendimento (telefônico e pessoal). No Sistema Integrado de Segurança Pública do Estado do Pará (SISP), por exemplo, são cadastrados cerca de mil boletins de ocorrência por dia.

Como existe uma grande quantidade de dados nas bases de dados dos órgãos de Segurança Pública, tem-se um grande potencial para explorar esses dados e transformálos em informação relevante. No entanto, na maioria das vezes, muitas informações são deixadas de serem abordadas, devido a carência de pessoas e ferramentas para trabalharem com esses dados.

Uma importe área em crescimento atualmente é a técnica de mineração de dados, que consiste em encontrar conhecimento implícito e não-trivial em grandes bases dados. Esta técnica permite encontrar relações entre as variáveis que são difíceis de serem executadas manualmente pelo ser humano. No entanto, para o objetivo deste trabalho, será abordada apenas a utilização de técnicas estatísticas incluindo apenas a análise descritiva, construção de gráficos, tabelas e mapas. Um exemplo da utilização de mineração de dados em Segurança Pública pode ser observado em Santos e Silva Filho (2007).

Apesar da grande quantidade de dados nas bases de dados, a próxima subseção aborda a necessidade do tratamento dos dados antes da aplicação das técnicas estatísticas.

\subsection{A Necessidade de Tratamento nos Dados}

Como discutido nas seções anteriores, existe uma grande quantidade de dados nas bases de dados das organizações. No entanto para trabalhar com esses dados, deve-se realizar um tratamento, a fim de eliminar informações inconsistentes, informações ausentes, a inclusão ou exclusão de alguns atributos, dentre outros. Trabalhar com os dados diretamente pode ocasionar em falta de confiabilidade nos resultados gerados.

Considerando o SIECRIM, por exemplo, que será melhor detalhado ao longo desse artigo, diversas transformações na base de dados do SISP foram realizadas a fim de que pudessem ser gerados os relatórios automaticamente diretamente de uma base de dados. Tal necessidade deu-se ao fato de que são colocados nos relatórios os valores com campos exatamente como estão descritos nas bases de dados. Surgindo a necessidade de uma análise cuidadosa na formatação dos atributos, eliminação dos registros inconsistentes e inclusão de novos campos a partir de outros.

Para armazenar os dados tratados para serem utilizados na geração automática dos relatórios, foi construído um data warehouse com as informações da criminalidade. Data warehouse é um repositório de informações para suportar decisões. Ele coleta dados a partir de diversas aplicações de uma organização, integra os dados em áreas lógicas de assuntos dos negócios, armazena a informação de forma que ela fique 
acessível e compreensível a pessoas não técnicas responsáveis pela tomada de decisões [Pazzani \& Mani, 1997].

A necessidade de tratamento de dados aumenta consideravelmente, na medida em que se necessita integrar diferentes bases de dados em um repositório central. A importância da integração será discutido na próxima seção.

\subsection{A Importância da Integração de BD}

Embora seja possível, o acesso às informações das enormes bases de dados, tem-se uma grande dificuldade referente a não-integração das bases de dados dos órgãos de Segurança Pública, o que dificulta sobremaneira o estabelecimento de um "retrato fiel" da situação da criminalidade, além de gerar informações duplicadas e com modelos de relatórios diferentes.

A interoperabilidade e a integração de sistemas entre os órgãos aumentam a qualidade, consistência, confiabilidade e acessibilidade às informações. Em consequiência disso, aumenta-se também a qualidade das decisões, tendo em conta a eliminação na redundância dos dados e a propensão ao que o erro implica. Adicionalmente, pelo compartilhamento de dados entre sistemas, a interoperabilidade e a integração melhoram o tempo de acesso à informação, fator crítico do processo decisório [Roberts, 2001].

Com a integração das bases de dados, pode-se ter a elaboração de relatórios mais completos e com maior confiabilidade. Porém, tem-se a desvantagens de uma maior complexidade para a construção desse repositório central e a necessidade da eliminação de informações redundantes e inconsistentes.

\section{SIECRIM: Sistema Estatístico Criminal}

Nesta seção será apresentado o Sistema Estatístico Criminal (SIECRIM), desenvolvido pelo Grupo de Estudos em Pesquisas Estatísticas e Computacionais (GEPEC) em parceria com o Governo do Estado do Pará.

\subsection{Contexto}

Os órgãos de gestão da Segurança Pública brasileira ainda não possuem recursos e instrumentos de tecnologia do conhecimento e da informação disponíveis, especialmente quando se trata de produtos da análise criminal baseados em estatísticas computadorizadas e SIG (Sistemas de Informações Geográficas) [Dantas, 2004].

Neste sentido, buscou-se desenvolver um sistema para automatizar a geração dos relatórios estatísticos, conhecido como SIECRIM, com o objetivo principal de auxiliar a análise criminal. O Sistema Estatístico Criminal (SIECRIM) foi projetado para proporcionar rapidez na produção e disponibilização das informações a respeito da criminalidade. Essa rapidez não é garantida somente pela automatização da geração dos relatórios, mas também pelo fato do sistema está disponível para usuários cadastrados interessados em obter informações da criminalidade através da internet.

O principal diferencial é a automatização desses relatórios e a utilização de georeferenciamento para proporcionar noções de distância e proximidades que são importantes para a identificação do local onde os crimes acontecem, assim como a intensidade dos mesmos. Os relatórios gerados por este sistema são automatizados e 
produzem análises descritivas das tabelas e gráficos estatísticos, além da representação da informação na forma de mapas. Essas análises, tabelas e gráficos foram discutidas junto aos usuários, com o apoio de pesquisadores estatísticos.

\subsection{Arquitetura do Sistema}

O SIECRIM foi desenvolvido com plataformas de desenvolvimento baseados em software livre. O sistema de gerenciamento de banco de dados (SGBD) utilizado foi o PostgreSQL, sendo que uma das razões para a adoção deste SGBD é o fato dele tratar as informações geográficas, necessárias para a geração dos mapas automáticos. Para mais detalhes, consultar Ribeiro \& Moreira, 2007. A linguagem de desenvolvimento utilizada para implementar este sistema foi a linguagem $J A V A$, que possui as vantagens de ser multiplataforma, portável, dentre outros. O servidor de Mapas utilizado foi o GeoServer e o ambiente utilizado para modelar os relatórios foi o Ireport.

Um aspecto a ser destacado na arquitetura é a necessidade de flexibilidade quanto à tecnologia utilizada para a implementação dos relatórios. O pacote Report, como mostrado na Figura 1, foi projetado considerando essa necessidade, já que a sua função é gerar os relatórios. Desta forma, projetou-se a interface ReportManager que define os métodos a serem implementados para a visualização de relatórios. Sendo assim, o SIECRIM pode visualizar os relatórios utilizando diferentes bibliotecas que implementem essa funcionalidade ou mesmo substituí-las sem grandes impactos ao sistema.

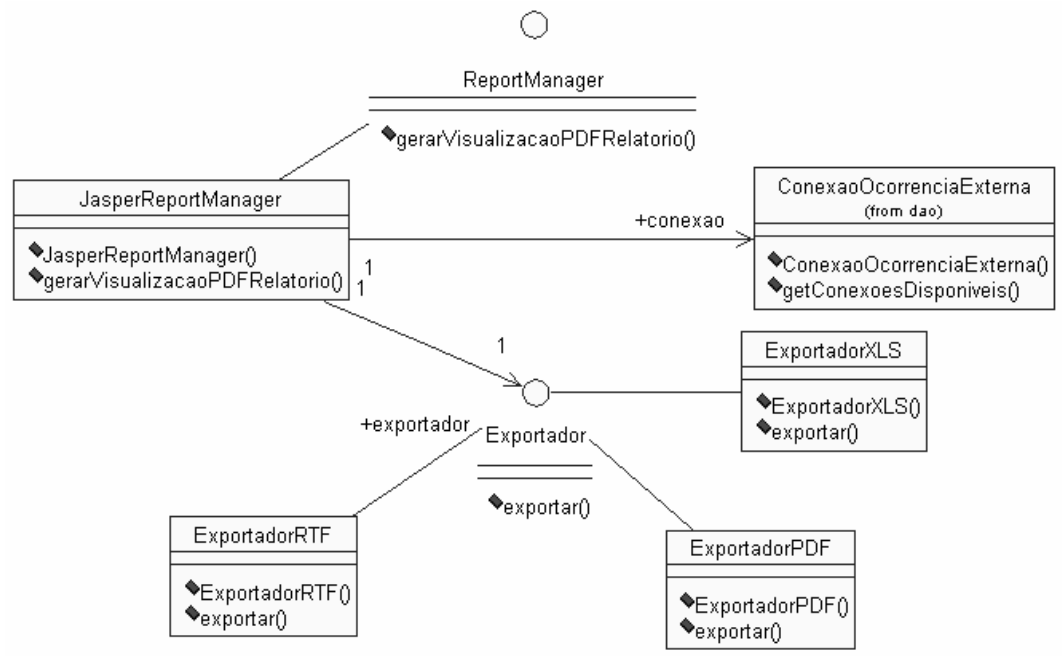

Figura 1. Diagrama de Classes do Pacote Report

Outro aspecto importante a ser mencionado é a possibilidade da integração de outras bases de dados como a do Detran (Departamento Nacional de Trânsito), SUSIPE (Superintendência do Sistema Penitenciário do Estado), dentre outros. Fato este que possibilita que a geração automática dos relatórios seja estendida para outras bases de dados da Secretaria de Segurança Pública.

Para suprir essa necessidade, fez-se a distinção entre o acesso ao banco de dados interno e externo (classes DAOInterno e DAOExterno, respectivamnente), as quais possuem classes distintas para conectar com seus respectivos bancos de dados (classes ConexaoNativa e ConexaoOcorrenciaExterna). A classe ConfiguraçãoOcorrenciaExterna, é instanciada de acordo com as informações contidas 
em um arquivo de configuração do sistema, que possui todos os parâmetros necessários para a conexão com qualquer banco de dados (endereço do servidor, login, senha, nome do driver do banco de dados a ser utilizado), bem como as informações de mapeamento da estrutura de tabelas do banco de dados externo para o formato de ocorrência definido no SIECRIM. As classes supracitadas estão destacadas no diagrama de classes deste pacote, o qual é mostrado logo abaixo na Figura 2.

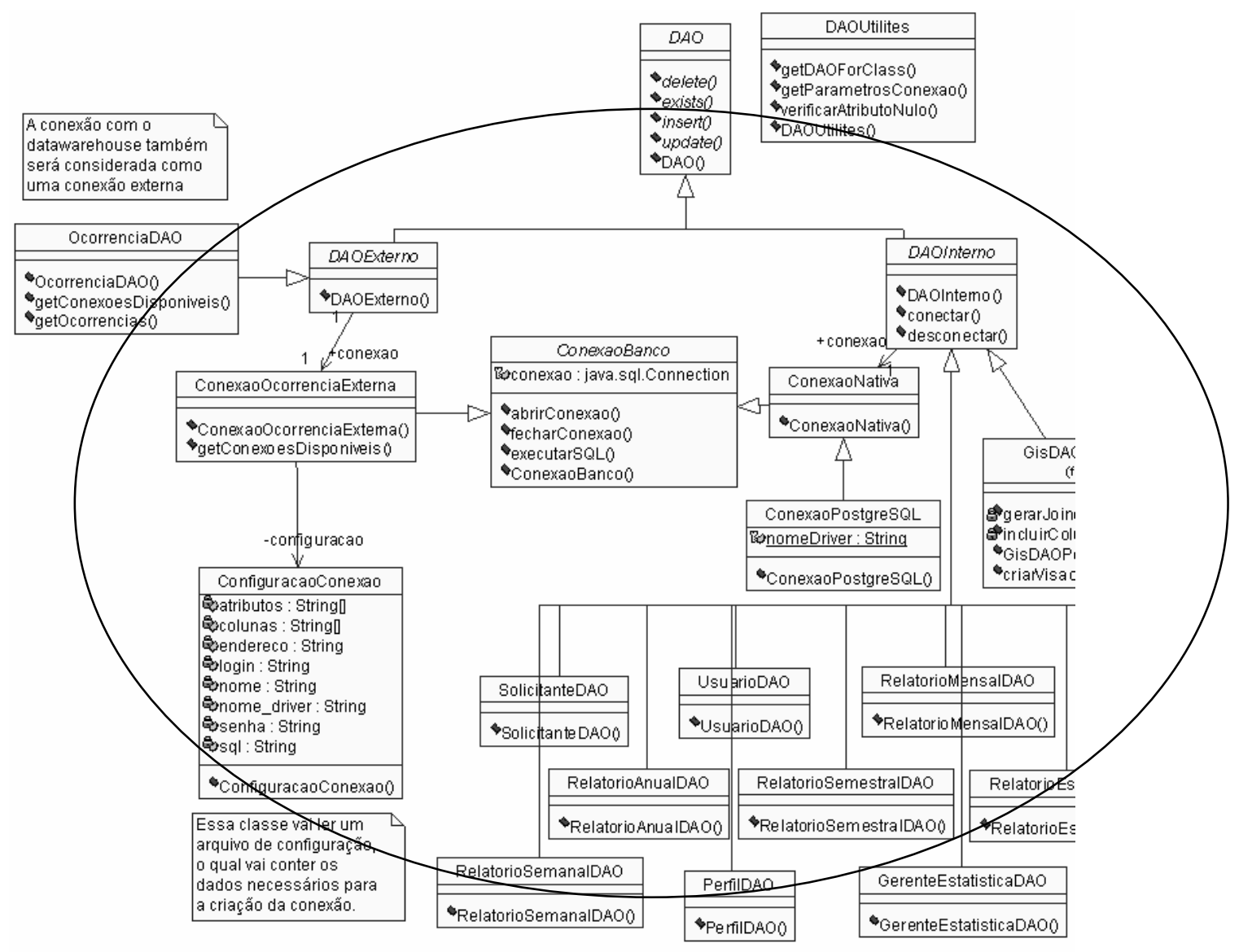

Figura 2. Diagrama de Classes do Pacote DAO

\subsection{Funcionamento do Sistema}

Para utilizar a principal funcionalidade do sistema, que consiste na geração automática de relatórios, o usuário deverá realizar o login no sistema. Uma vez logado, o usuário deverá seleciona a opção de geração de relatórios, e em seguida, conforme observado na Figura 3, selecionar o tipo de relatório desejado (1). Além disso, o sistema disponibiliza um conjunto de parâmetros que podem ser ajustados conforme as necessidades do usuário, como a escolha de quais delitos (4), zona de policiamento (ZPOL) (2) e período (3) serão abordados no relatório, fornecendo dessa forma uma grande flexibilidade em sua geração.

Após a definição dos parâmetros, o usuário do sistema pode optar por gerar o relatório em dois diferentes formatos: PDF ou RTF (5). Este último formato pode ser editado conforme o interesse do usuário. Dessa forma os Relatórios são disponibilizados na WEB onde cada usuário possui um perfil com privilégios diferentes, ou seja, cada 
Seccional ou ZPOL tem acesso ao sistema podendo, dessa forma, ter acesso aos índices de criminalidade de suas respectivas áreas.

O sistema cadastra os Relatórios, bem como mantêm o status (em aberto, finalizado, etc.) de cada relatório. Dentre outras funcionalidades, o usuário do sistema pode adicionar informações à base de dados, cadastrar solicitação de relatório esporádico ou exportar relatórios.

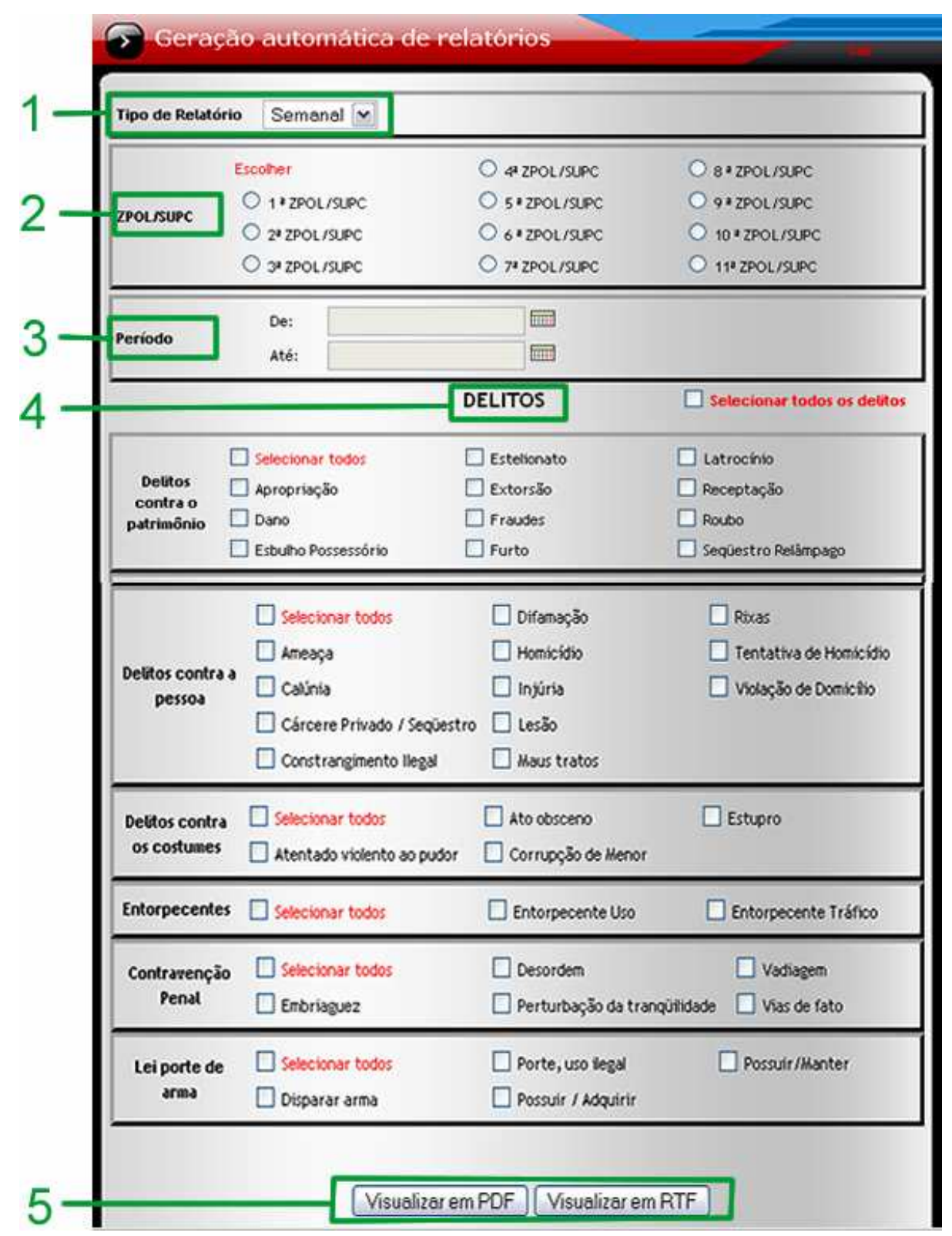

Figura 3 - Interface para selecionar os parâmetros de geração do relatório

Pode-se observar a partir da Figura 4, que os relatórios gerados pelo SIECRIM abordam análises estatísticas dos crimes registrados na RMB, que servem de base para o processo de formulação e implementação de estratégias, com o objetivo de subsidiar os 
órgãos responsáveis pela Segurança Pública no Estado do Pará. Atualmente o sistema disponibiliza a geração do modelo de relatório Semanal e Mensal.

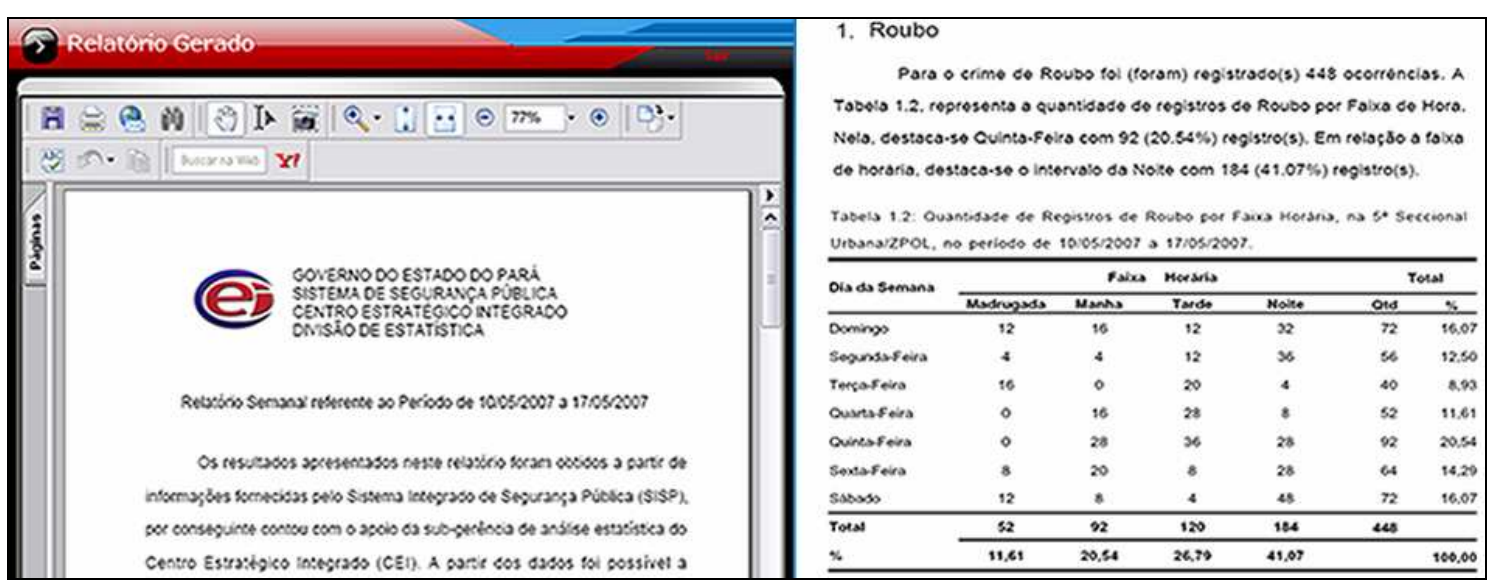

Figura 4 - Interface do Relatório Gerado e tabela com análise descritiva

As análises estatísticas são elaboradas com base em tabelas, gráficos e análises descritivas do comportamento da criminalidade e da violência, na RMB. Na Figura 5 observa-se um gráfico de pizza, dois gráficos de linha, e um gráfico de barras que são gerados automaticamente pelo sistema.

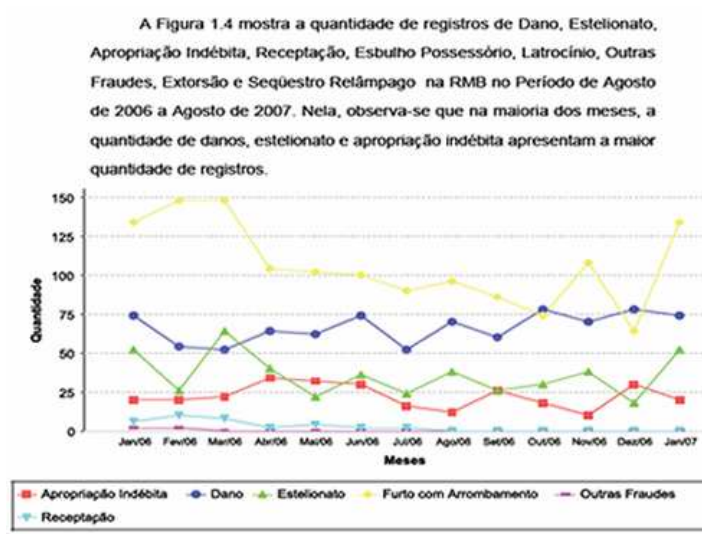

Figura 1.1: Percontual do Registros de Roubo por faica horaria, na 5 * Seccional Urbana/ZPOL, no poriodo do 10/05/2007 a 17/05/2007.

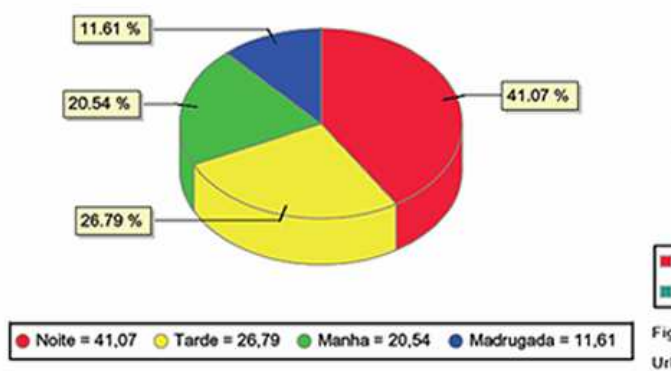

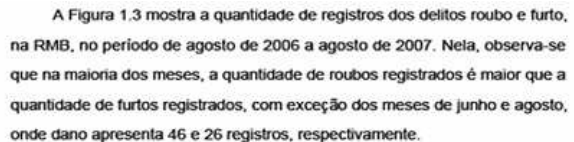

onde dano apresenta 46 e 26 registros, respectivamente.

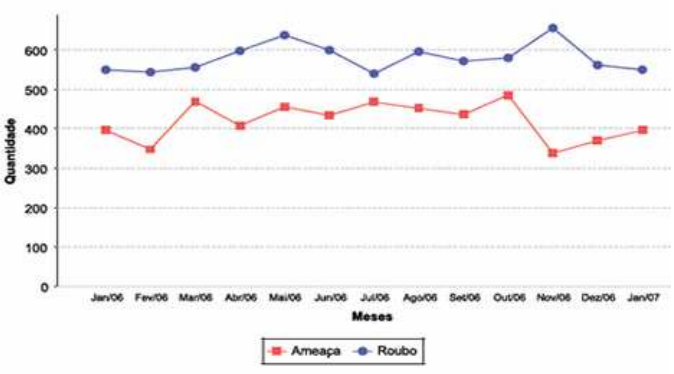

Poxcontagem

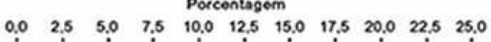

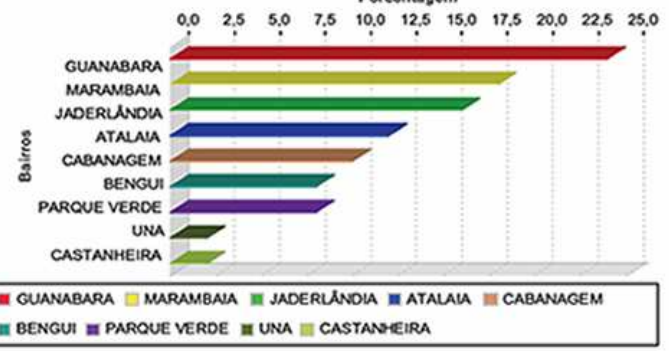
I BENGUI \# PNRQUE VERDE \# UNA II CASTANTEIRA

Figura 5 - Gráficos e analises descritivas dos relatórios

Além disso, conforme observado na Figura 6, o sistema contempla a geração automática de mapas visando aumentar a forma de interpretação das ocorrências. Diante do avanço da criminalidade em sua atuação e estratégia na sociedade, a técnica de Georeferenciamento se torna uma ferramenta importante no combate e prevenção de 
crimes, onde através desta, busca-se visualizar as informações em mapas digitalizados e com base nessas informações, tomar as devidas decisões gerenciais.

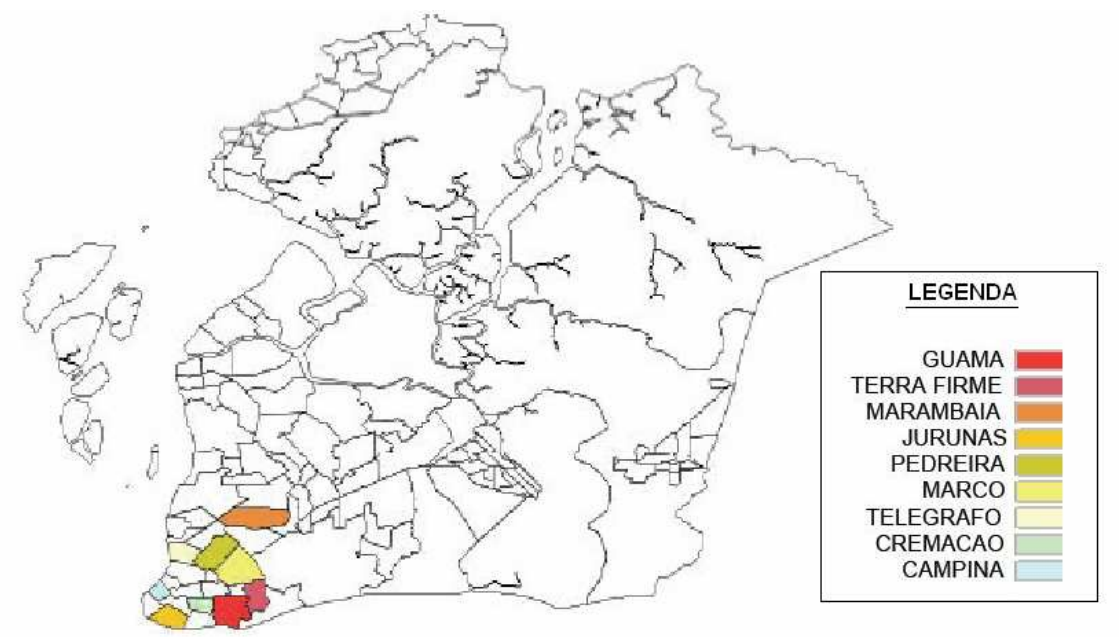

Figura 6 - Exemplo de mapa gerado pelo SIECRIM para os crimes mais registrados nos bairros da RMB, no ano de 2006. Fonte: CEI.

Futuramente, o SIECRIM visa implementar a geração de Alarmes de Segurança, cujo objetivo consiste em avisar imediatamente as autoridades de Segurança Pública quando um determinado índice de criminalidade estiver fora do padrão de normalidade. Além disso, visa também implementar uma interface mais amigável para a realização de consultas no SISP, uma vez que a interface atual não atende completamente as necessidades dos usuários.

Outra funcionalidade pretendida consiste na utilização de técnicas de Mineração de Dados e de Mineração de Textos na busca de características, regras e regularidades em um grande conjunto de dados, além de pesquisa de palavras chave nos Boletins de Ocorrência Policial.

\section{Trabalhos relacionados}

Com o intuito de contextualizar o ambiente em que o Sistema Estatístico de Criminalidade (SIECRIM) se encontra, será mostrados a seguir, alguns sistemas de informação, de caráter público, ilustrando algumas perspectivas do desenvolvimento destes sistemas, que como o SIECRIM, realiza a geração automática de relatórios e desta forma, oferecem suporte à tomada de decisão.

Atualmente no Brasil, existem poucos sistemas de informação que implementam funcionalidades semelhantes às funcionalidades do SIECRIM. Como por exemplo, o georeferenciamento da informação, que é algo que exige certo nível de complexidade além de outras funcionalidades que serão agregadas durante o desenvolvimento do sistema.

Celepar (2007) destaca o Sistema de Defesa e Proteção ao Consumidor. Este sistema é uma ferramenta que permite registrar e acompanhar os atendimentos efetuados pelo PROCON e fornecer relatórios, estatísticos e gerenciais, para avaliação e direcionamento de ações relacionadas aos direitos do cidadão. Foi concebido pelo governo do Estado do Paraná, sendo, assim como o SIECRIM, um sistema Web. 
Assim como o SIECRIM, o Sistema de Defesa e Proteção ao Consumidor implementa a geração automática de relatórios. No entanto, os relatórios são voltados aos atendimentos como: fornecedor mais reclamado, assunto mais reclamado, reclamações de um fornecedor, atendimentos de um consumidor, dentre outros. No entanto, este sistema foi desenvolvido utilizando ferramentas proprietárias: Active Server Pages (ASP) e SQLServer. O que provavelmente aumentou consideravelmente o custo final do produto.

Outro sistema que implementa a geração automática de relatórios é o Sistema de Informação Socioeconômica do Município de São Paulo (SISEM). Sua principal função é agrupar em um único ambiente computacional, informações relevantes para a administração do município e de suas secretarias e órgãos, configurando-se como instrumento efetivo de gestão (Prefeitura.SP, 2007).

É um projeto de Inteligência de Gestão, onde informações antes isoladas passam a ser associadas, consolidadas e atualizadas servindo como instrumento de diagnóstico da realidade local, subsidiando a definição de diretrizes e tomadas de decisão do governo. O SGBD utilizado como base desse sistema também é o SQLServer. A geração de relatórios é realizada utilizando um dos componentes do pacote Microsoft Office (Excel).

\section{Considerações Finais}

O SIECRIM foi implementado com o objetivo de automatizar a geração dos relatórios, trazendo rapidez para a produção e disponibilização dessas informações. O principal diferencial desse sistema é a utilização de georeferenciamento para proporcionar noções de distância e proximidades que são importantes para a identificação do local onde os crimes acontecem, assim como a intensidade dos mesmos.

Por outro lado, a integração das bases de dados facilita a geração dos relatórios estatísticos referentes à criminalidade no estado do Pará, permitindo que informações únicas sejam produzidas, com maior velocidade, e que sirvam para a tomada de decisões e de estratégias de todos os órgãos componentes do sistema de Segurança Pública do Estado do Pará.Com base nessa compreensão do ambiente externo à organização, o plano estratégico é uma ferramenta para auto-organização, isto é, de mudança e ampliação das capacidades, processos e resultados, visando melhorar a eficiência e eficácia das tomadas de decisão.

Dessa forma, o SIECRIM vem beneficiando a tomada de decisão na Segurança Pública deixando de gerar manualmente os Relatórios, proporcionando a automatização desse processo.

\section{Referências}

CELEPAR - Cia de Informática do Paraná - Administração de Conteúdo http://www.celepar.pr.gov.br/modules/conteudo/conteudo.php?conteudo=23. Acessado em 2007.

Dantas, G. F. L., e Souza, N. G. As bases introdutórias da análise criminal na inteligência policial, 2004.

Gottlieb, S.L., Arenberg, S., e Singh, R., 2002. Crime Analysis: from first report to final arrest: study guide and wordbook. Montclair, CA: Alpha Publishing, p. 5. 
Mizzaro, Stefano. Relevance: The Whole History. Journal of the American Society for Information Science, New York: John Wiley \& Sons. v.48, n.9, p.810-832. 1997.

Orlandini, Leandro. A importância dos Sistemas de Informação. Disponível em http://www.bonde.com.br/colunistas/colunistasd.php?id_artigo=1646. Acessado em Setembro de 2007.

Pazzani, M.; Mani S. ; Comprehensible knowledge discovery in databases. In Proceedings of the Nineteenth Annual Conference of the Cognitive Science Society, Lawrence Erlbaum, 1997. pp. 596-601.

Prefeitura.SP - SISEM - SISTEMA DE INFORMAÇÕES SOCIOECONÔMICAS http://portal.prefeitura.sp.gov.br/secretarias/governo/sisem/0001. Acessado em 2007.

Ramos, E. M. L. S.; Carvalho, D.C.O. ; Queiroz, J. C. B. Um Estudo da Série Histórica da Quantidade de Registros de Roubos na Região Metropolitana de Belém no Período de Janeiro de 1994 a Dezembro de 2006. 12a Escola de Séries Temporais e Econometria, 2007, Gramado/RS, Brasil.

Ribeiro, T. V., Moreira, P. D. O. Arquitetura de um SmallSIG para apoio ao Planejamento Estratégico na Área de Segurança Pública, artigo publicado na Conferencia Latinoamericana de Informática(CLEI) 2007.

Roberts, D. J. Integration in the context of justice information systems: a common undestanding., Sacramento, CA - USA, SEARCH - The Nacional Consortium for Justice Information and Statistics. 2001.

Santos, E. L. S. O Sistema de Levantamento Estatístico para a Análise Criminal na Polícia Militar no estado do Mato Grosso. Curso de Aperfeiçoamento de Oficiais. Cuiabá. 2002.

Santos, F.H.M.; Silva Filho, L.A. Utilização de Sistemas de Informação para Apoiar a Tomada de Decisão na Segurança Pública do Estado do Pará. Trabalho de Conclusão de Curso. Belém. UFPA. 2007. 